\title{
Glucose intolerance and dyslipidaemias in persons with paraplegia and tetraplegia in South India
}

\author{
George Tharion, K Rajendra Prasad, Lata Gopalan and Suranjan Bhattacharji \\ Department of Physical Medicine and Rehabilitation, Christian Medical College Hospital, Vellore, 632 004, India
}

\begin{abstract}
Glucose intolerance and dyslipidaemias have been reported among paraplegics and tetraplegics. In this study we determined the lipid profiles and the glucose tolerance in a group of 48 persons with spinal cord lesions who had been rehabilitated in our Department of Physical Medicine and Rehabilitation. This non-randomised, descriptive study was conducted as part of the annual medical follow-up of these individuals. Hypertension was observed in only $6 \%$ of the subjects. Fasting hyperglycemia was observed in $19 \%$ and glucose intolerance in $23 \%$ of the subjects. Total cholesterol was abnormal in $2 \%$, but $58 \%$ had low levels of high density lipoprotein (HDL). 10\% of the subjects had raised low density lipoprotein (LDL) levels. The cardioprotective HDL fraction may remain very low even while the total cholesterol level is within normal limits, and unless a lipid profile estimation is done, this group of individuals who are at cardiovascular risk may remain undetected. We conclude that glucose intolerance and dyslipidaemias are common among paraplegic and tetraplegic individuals, and these metabolic derangements may contribute to increased cardiovascular morbidity.
\end{abstract}

Keywords: paraplegia; tetraplegia; cholesterol; HDL cholesterol; glucose intolerance; dyslipidaemias

\section{Introduction}

Life expectancy following spinal cord lesions is improving with better initial care, comprehensive rehabilitation and efficient management of life threatening complications such as urinary tract infection and pressure sores. Such better survival has challenged the medical profession to evaluate their risk factors which would predict life expectancy and cardiovascular morbidity.

The incidence of cardiovascular disease is reported to be higher in the spinal cord injury population. ${ }^{1}$ Traditionally elevated serum cholesterol and high blood pressure were considered as high risk factors for coronary heart disease. ${ }^{2,3}$ The cardioprotective role of high density lipoprotein (HDL) and its subfractions have been established earlier and it is now suggested that increased attention should be given to HDL cholesterol levels not only when the serum cholesterol level is high as recommended by the National Cholesterol Education Programme, but also when the total cholesterol level is not elevated. ${ }^{4,6}$ Fasting hyperglycaemia and impaired glucose tolerance have been reported in subjects following spinal cord injury. ${ }^{7}$ Both carbohydrate metabolism ${ }^{8}$ and level of physical activity $^{9}$ have each, separately, been shown to influence the HDL levels, and hence we decided to

Correspondence: G Tharion study the serum lipid profile and the glucose tolerance among rehabilitated paraplegic and tetraplegic persons.

\section{Materials and methods}

One hundred and thirty-eight subjects with chronic spinal cord lesions who had been rehabilitated in our department were invited to come for an annual medical follow up in February, 1995. Fifty-two subjects came, and of these, 48 subjects fulfilled the inclusion criteria which were:- (1) Adults with paraplegia or tetraplegia; (2) Those with lesions of more than one year duration; (3) Those without complications such as symptomatic urinary tract infection, renal failure and pressure ulcers.

A clinical evaluation including history of smoking, walking status and estimation of blood pressure, was done. A diastolic blood pressure above $90 \mathrm{mmHg}$ and a systolic blood pressure above $140 \mathrm{mmHg}$ were considered as a diagnosis of hypertension. Subjects who were using orthoses and crutches for ambulation were considered as walkers, and those who were dependent on wheelchairs or tricycles for their mobility were considered as non-walkers. Blood samples were taken after overnight fasting for the estimation of plasma glucose and for a serum lipid profile. Serum creatinine, packed cell volume and serum albumin were simulta- 
neously estimated to evaluate the general health status of these subjects. They were then given $75 \mathrm{gm}$ of glucose orally and blood was sampled for plasma glucose after a $2 \mathrm{~h}$ interval. The plasma glucose was estimated using the glucose oxidase - peroxidase (GOD) technique and lipid profile was estimated using cholesterol oxidase paraminophenazone (CHOD PAP) techniques. ${ }^{10,11}$ (The Bio-chemistry department of our Institution regularly takes part in the External Quality Assessment Schemes (EQAS) conducted by (i) Wolfron EQA Laboratory, PO Box 3909, Birmingham, B15 2UE, UK Lab No 3170 and (ii) Victoria Infirmary, Glasgow, Lab No 10). Fasting plasma glucose above $6.1 \mathrm{mmol} / \mathrm{L}$ and plasma glucose levels above $7.7 \mathrm{mmol} /$ $\mathrm{L}$ at $2 \mathrm{~h}$ following the oral glucose load were considered abnormal. The lipid profile values were considered in different groups as given in Table 1 as suggested by the National Cholesterol Education Programme. 3 Thulaseedharan et al has reported similar lipid profile values in 173 normal healthy South Indian adults. ${ }^{5}$

\section{Results}

Among the 48 subjects studied, there were 43 males and five females. Three subjects had cervical cord lesions, 28 thoracic and 17 lumbar cord lesions. The age of the subjects ranged between $16-52$ years; 16 were less than 30 years of age and 32 were between 30 and 60 years of age. The interval after the onset ranged from 1-20 years; 25 subjects were paralysed for a period of less than 5 years duration, 14 from $5-10$ years duration and nine for more than 10 years duration. There were 27 walkers and 21 non-walkers among this group.

On clinical evaluation the blood pressure was found to be elevated in three subjects. All of the subjects had normal serum creatinine levels (below $132.6 \mu \mathrm{mol} / \mathrm{L}$ ), normal serum albumin levels $(35-55 \mathrm{~g} / \mathrm{L})$ and normal packed cell volumes (above 30\%). Among the 48 subjects evaluated, nine $(19 \%)$ had fasting hyperglycemia. Following a challenge with oral glucose 11 subjects $(23 \%)$ had hyperglycemia at $2 \mathrm{~h}$. Total serum cholesterol was above $6.21 \mathrm{mmol} / \mathrm{L}$ in one subject $(2 \%)$. The HDL cholesterol levels were above $0.90 \mathrm{mmol} / \mathrm{L}$ in $20(42 \%)$ subjects; between $0.77-$ $0.88 \mathrm{mmol} / \mathrm{L}$ in $11(23 \%)$ and below $0.77 \mathrm{mmol} / \mathrm{L}$ in 17 (35\%). The Low Density Lipoproteins (LDL) levels were below $3.36 \mathrm{mmol} / \mathrm{L}$ in $43(90 \%)$; between $3.36-$ $4.14 \mathrm{mmol} / \mathrm{L}$ in $5(10 \%)$ and none had levels above $4.14 \mathrm{mmol} / \mathrm{L}$. Ten subjects $(21 \%)$ had serum triglyceride levels above $2.26 \mathrm{mmol} / \mathrm{L}$ (refer Table 2).

The mean fasting plasma glucose of the subjects was found to be significantly higher and the mean total cholesterol, HDL and LDL levels significantly lower than normal (refer Table 3). Among the 16 subjects below 30 years of age eight had low HDL (50\%) and among 32 subjects above 30 years of age 20 had low HDL values $(62.5 \%)$. Among the 27 walkers 15 had low HDL (55.5\%) and among the 21 non-walkers 13
Table 1 Normal borderline and high risk lipid profile groups

\begin{tabular}{llcl}
\hline & $\begin{array}{c}\text { Normal } \\
\text { mmol } / L\end{array}$ & $\begin{array}{c}\text { Borderline } \\
\mathrm{mmol} / \mathrm{L}\end{array}$ & $\begin{array}{c}\text { High risk } \\
\mathrm{mmol} / \mathrm{L}\end{array}$ \\
\hline Total cholesterol & $<5.18$ & $5.18-6.19$ & $>6.21$ \\
LDL cholesterol & $<3.36$ & $3.36-4.11$ & $>4.14$ \\
HDL cholesterol & $>0.90$ & $0.77-0.88$ & $<0.77$ \\
Triglycerides & $<2.26$ & $2.26-4.52$ & $>4.52$ \\
\hline
\end{tabular}

Table 2 Distribution of patients in the normal borderline and high risk lipid profile groups

\begin{tabular}{llcc}
\hline & Normal & Borderline & High risk \\
\hline Total cholesterol & $44(92 \%)$ & $3(6 \%)$ & $1(2 \%)$ \\
HDL cholesterol & $20(42 \%)$ & $11(23 \%)$ & $17(35 \%)$ \\
LDL cholesterol & $43(90 \%)$ & $5(10 \%)$ & - \\
Triglycerides & $38(79.1 \%)$ & $8(16.7 \%)$ & $2(4.2 \%)$ \\
\hline
\end{tabular}

Table 3 Distribution of plasma glucose and lipid profile in the study sample

\begin{tabular}{lccc}
\hline & \multicolumn{3}{c}{ Mean } \\
& $\mathrm{n}$ & $($ mmol $/ L)$ & s.d. \\
\hline Fasting Plasma glucose & 48 & 5.61 & 0.97 \\
Plasma glucose 2 h after 75 gm glucose & 48 & 6.17 & 1.74 \\
Total cholesterol & 48 & 3.76 & 0.87 \\
HDL cholesterol & 48 & 0.85 & 0.20 \\
LDL cholesterol & 48 & 2.09 & 0.62 \\
Triglycerides & 48 & 1.73 & 1.00 \\
\hline
\end{tabular}

had low HDL levels $(62 \%)$. There was no correlation between the HDL levels and the walking status.

\section{Discussion}

An increased incidence of hypertension and ischemic heart disease among SCI subjects has been reported. The inverse relationship between the HDL cholesterol level and coronary heart disease has been established by earlier studies. Bauman et $a l^{12}$ Krum et $a l^{13}$ and Brenes et $a l^{14}$ have reported depressed serum HDL cholesterol levels in chronic spinal cord injury. However, Cardus et al, ${ }^{15}$ reported that subjects with SCI do not have higher levels of total cholesterol, LDL or lower levels of HDL. These conflicting reports prompted us to evaluate the lipid profile status and glucose tolerance among subjects with spinal cord injury in South India. This study was undertaken as a part of the annual medical follow up of all patients with spinal cord lesions who were rehabilitated by our department who live within $100 \mathrm{~km}$ from our Institution. Although we invited 138 patients to participate, only 52 patients came for the follow up. The patients who could not come to the hospital have 
been followed up by home visits conducted by our Social Worker along with the medical team. Although we could not evaluate their lipid profile and glucose tolerance, they were found to be surviving in the community and were not more handicapped than were those who participated in the follow-up clinic. The reasons given for non-attendance were that they did not feel the need for check up, or experienced difficulty in arranging transport and carers to come to the Institution. Thus only 52 patients could be evaluated among which 48 fulfilled the inclusion criteria.

In this study all subjects had normal packed cell volumes, serum albumin and creatinine values, thus establishing their general health status to be satisfactory. ${ }^{16}$ Hypertension was found in $6 \%$ in contrast to the prevalence of $24 \%$ reported by Yerkutiel et al. Fasting hyperglycaemia was observed in $19 \%$ and impaired glucose tolerance in $23 \%$ of the subjects, similar to Duckworth et al who have reported fasting hyperglycaemia in $23 \%$ and impaired glucose tolerance in $40 \%$ of their subjects. The total cholesterol was elevated only in $2 \%$ of the subjects as found by others. None of our subjects had LDL levels above $4.14 \mathrm{mmol} / \mathrm{L}$ to be classified in the high risk LDL cholesterol group, similar to that reported by Cardus et $a l$, but different from Bauman et al who reported high risk LDL levels in $18 \%$ of his patients. The HDL levels were found to be less than $0.9 \mathrm{mmol} / \mathrm{L}$ in $58 \%$ of our subjects, similar to that reported by Bauman et al, Krum et al and Brenes et al. The lipid profiles did not show any correlation between the neurological level, duration since injury or walking status. The serum triglycerides levels, even though not an independent coronary artery disease risk factor, was found to be elevated in $21 \%$ of subjects. Stampfer et al have reported that the HDL level is a powerful predictor of coronary heart disease risk and this inverse association is stronger for men with lower levels of total cholesterol. The general recommendation to analyse the complete lipoprotein profile only if the cholesterol is elevated above $6.47 \mathrm{mmol} / \mathrm{L}$ may result in this group of individuals at risk, with low total cholesterol and low HDL, being undetected.

The reduced physical activity levels in persons with spinal cord lesions, makes them an important group to determine the potentially detrimental effects of inactivity. The impaired carbohydrate metabolism in addition to the reduced physical activity could be contributing to the dyslipidaemia that is prevalent in this population with paraplegia and tetraplegia and further research may provide insight into the causes of these metabolic derangements and cardiovascular disease.

\section{References}

1 Yerkutiel $\mathrm{M}$ et al. The prevalence of hypertension, ischaemic heart disease and diabetes in traumatic spinal cord injured patients and amputees. Paraplegia 1989; 27: 58-62.

2 Multiple Risk Factor Intervention Trial Research Group Multiple Risk Factor Intervention Trial: risk factor changes and mortality results. JAMA 1982; 248: $1465-1476$.

3 Report of the National Cholesterol Education Program Expert Panel of Detection, evaluation and treatment of High Blood Cholesterol in Adults. Arch Intern Med 1988; 148: 36-69.

4 Summary of the Second Report of the National Cholesterol Education Program (NCEP) Expert Panel on Detection, Evaluation, and Treatment of High Blood Cholesterol in Adults (Adult Treatment Panel II). JAMA 1993; 269 No. 23: 30153023.

5 Thulaseedharan N, Augusti KT. Risk factors for coronary heart disease in non-insulin dependant diabetes mellitus. Indian Heart Journal 1995; 47: 471 - 76 .

6 Stampfer MJ et al. A prospective study of cholesterol, apoliopoprotiens, and the risk of myocardial infarction. $N$ Eng J Med 1991; 325: 373-381.

7 Duckworth WC, Jallepalli P, Solmon SS. Glucose Intolerance in Spinal Cord Injury. Arch Phys Med Rehabil 1983; 64: 107-110.

8 Golay A et al. High Density Lipoprotein (HDL) metabolism in non-insulin dependent diabetes mellitus: measurement of HDL turnover using tritiated HDL. J Clin Endocrinol Metab 1987; 65: $512-518$.

9 La Porte RE et al. HDL cholesterol across a spectrum of physical activity from quadriplegia to marathon running. Lancet $1983 ; 1$ : $1212-1213$

10 Siedal J, Hagele EO, Ziegenhorn J, Wahlefeld AW. Reagent for the enzymatic determination of serum total cholesterol with improved lipolytic efficiency. Clinical Chemistry 1983; 29: 10751080.

11 Kattermann R et al. J Clin Chem Clin Biochem 1984; 22(3): 245 51 .

12 Bauman WA et al. Depressed serum high density lipoprotein cholesterol levels in Veterans with spinal cord injury. Paraplegia 1992; 30: $697-703$.

13 Krum $\mathrm{H}$ et al. Risk factors for cardiovascular disease in chronic spinal cord injury patients. Paraplegia 1992; 30: $381-388$.

14 Brenes $G$ et al. High density lipoprotein cholesterol concentration physically active and sedentary spinal cord injured patients. Arch Phys Med Rehabil 1986; 67: 445-450.

15 Cardus D, Cardus FR, McTaggart BS. Lipid profiles in spinal cord injury. Paraplegia 1992; 30: $775-782$.

16 Glenn MB et al. Serum Albumin as a predictor of course and outcome on a Rehabilitation service. Arch Phys Med Rehabil 1985; 66: $294-297$. 